\title{
Laser Actuated Non-Invasive Smart Instrumentation - Enabling Lab-on-Chip
}

\author{
N. Z. Azeemi ${ }^{a^{*}}$, N. Ahmed ${ }^{b}$, N. U. Saquib ${ }^{c}$ \\ ${ }^{a}$ IEEE Council Member for Nano Materials, School of Research and Innovation, Skyline University College, Sharjah Academic City, UAE \\ ${ }^{b}$ M365 Security Group, Microsoft, Massachusetts, USA \\ ${ }^{c}$ Ministry of National Health Services, Regulation \& Coordination, Government of Pakistan, Pakistan \\ Corresponding author: "naeemazeemi@gmail.com
}

\begin{abstract}
Non-invasive optical instrumentation provides non-destructive, reliable, and precise control in industrial process regulation, especially when chemical compounds or organic material surfaces are always a point of care. Nanomaterial dynamics intrinsically exhibit higher order of visual scanning complexities, associate wholly or partially to the poor scanning instrumentations. Additionally, growing trends in analytical instrumentation towards smart Lab-On-a-Chip (IoT sensing nodes) have shifted the emphasis on sensitivity and robustness tailoring Product Specific Environment (PSE). This work presents a hybrid laser actuated scanning mechanism, rastered back and forth 3-D imaging technique enabling Microscopy to its widest application in biological and material sciences and hence rose challenge of predicting large missing or incorrect data obtained during experiments. Our Confocal Self Calibrated Interferometry based fabricated Laser Sensor demonstrates its efficacy in non-invasive scanning microscopy to achieve a highresolution 3D topographical view, eventually an add-on to the analytical model of microorganisms and nanomaterial. In contrast to linear controllers, PI controllers demonstrate better stability in controlling the laser leakage at tip, which consists of two channel tube adjustments and successively in laser reflector lens, Photo Multiplier Tube (PMT), and Data Acquisition Unit (DAU). We expose our results for error propagation across various grid patterns over a $1 \mathrm{~mm}^{2}$ section, plotting the intensity of a key band or bands over the PMT grid. We observe that the instrumentation errors can be nullified by modeling the ergodicity of information flow along with the SLM instrumentation.
\end{abstract}

Keywords - Smart instrumentation; IoT; non-invasive; laser; confocal; optical microscopy.

Manuscript received 18 Apr. 2020; revised 17 Mar. 2021; accepted 27 Apr. 2021. Date of publication 31 Oct. 2021. IJASEIT is licensed under a Creative Commons Attribution-Share Alike 4.0 International License.

\section{INTRODUCTION}

Industrial Process Regulation (IPR) is restrained tightly coupled scrutiny of scientific data and results in regulation of $\mathrm{R} \& \mathrm{D}$ for correct, up to date and conforms to the Regulation Bureau Service (RBS) indicators [1], [2]. In the same vein, they are desired to be both eco-friendly and exhibit low form factors to ever-increasing demand among smart urban areas. The increasing demand for smart instruments put forth additional decision indicators in the Instrument-in-Loop (IiL) to facilitate better Time-to-Deployment (ToD) in a given scenario [2]-[4], such as handheld measuring instrument. Working knowledge experts' important role expedites the transformation phase of an analytical model, while a comprehensive history of techniques and off-the-shelf devices augment the ToD. It eventually improves the proto development cycle, both in terms of cost-design time and the performance at both deployment and development latencies
[5]-[7]. The on-chip available cores add fast development pace, aligned with comprehensive design space exploration, which otherwise could lead to np-hard unless rapid prototyping is obtained, keeping all artificial intelligence machine learning engines in the development cycle. E.g., Qualcomm, Snapdragon 865 took three years to provide unprecedented functionality in bringing AI engines, $5 \mathrm{G}$ cores, cryptography on one core to support existing Cortex highperformance octa cores [8], [9]. However, the researchers did find its development history associated with Snapdragon 765 already available at various mobile platforms, including handheld instruments. Later on, a smart design cycle emerged into squeezing external data traffic onto SD 765, hence named SD 865 [8], [9].

This work brought together refined industrial instrumentation methodologies, tailoring smart industry requirements of the age, i.e., low form factor to accommodate OEM, and high-performance speed to meet the data traffic 
demand as generated in any sensing point on a typical IoT network [10], [11]. We achieved our objective, considering computing intensive non-linear analytical techniques that add another layer to the key industrial instrumentation. The miniaturization technology to $7 \mathrm{~nm}$ scale encourages us to tailor the demand in non-invasive instrumentation, which are widely used in scanning microorganisms both in vitro and ex vitro experiments and monitoring [12]-[14]. This conforms analytical observation and experience commenced by scientist, researcher or industrial use case lead into the miniaturization, hence termed as Lab-on-Chip (LoC); which not only evaluate a specimen comprehensively with a correct choice of instrumentation in place but also enable smart IoT devices subject to scenario constraints on appropriate applications [13], [15], [16]. We recommend a closed-loop decision tier for analytical method development based on three key characters, as shown in Fig. 1.

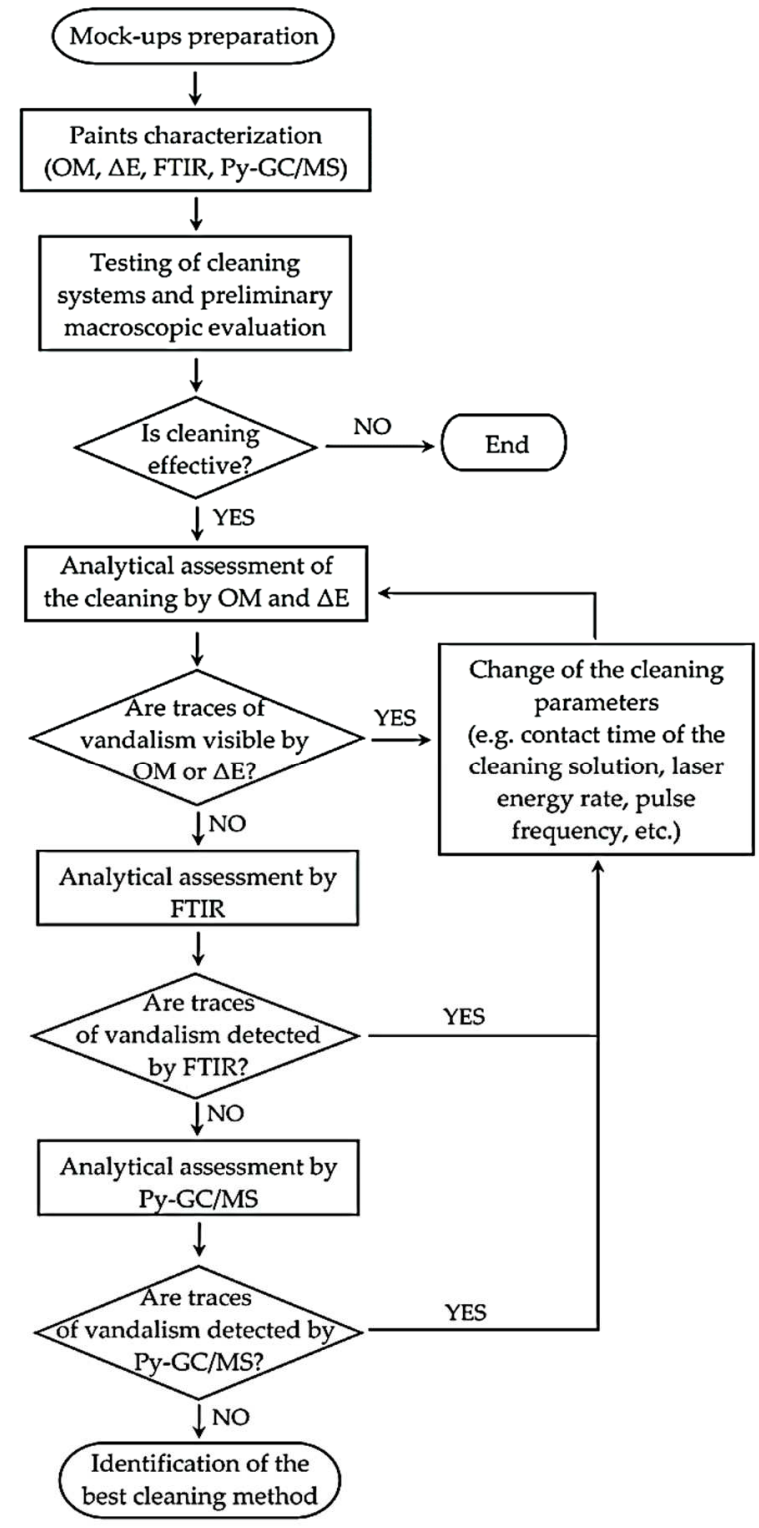

Fig. 1 Simplified Flowchart for Visualization and 3D Topographic Imagining Industrial Process Use Case [2]

An analyst is presumed to be aware of various available options (as expert knowledge or enabled with machine learning search engines), which enable them to reach an optimal desired solution in their provided scenario for offered design space $[17,18]$. The flexibility of such methodology is inherent in the virtualization of deployed technology history, enables a smart choice without compromising on successive learning, and eventually leads to a significant reduction in ToD.

\section{A. Non-Invasive Optical Illumination Analytics}

The specimen non-pervasive scanning is of common interest, especially researchers from diverse backgrounds as engineering, information management systems, pure sciences, who are involved in any capacity of analytical modeling from their observed and experimental logs [19]. Therefore, analytical methods-scientific development into theory, laws, and regulations can be witnessed among the academic profile to industrialists, edible items and their signature analysis, identification of toxic elements broadly addressed by the pharmaceutical industries in special and food-drug authorities. The critical per view of an analytical scientific experiment provide a multitude of dimension into any empirical model complement a vivid theoretical comprehension in contrast a rigorous hands-on laboratory experimental practices tailoring RBS [20, 21, 22]; hence provide reuse on any virtual platforms, as the term coined in IoT network sensing nodes. Each set of design space accessed in a multivariate optimization objective brings both principles lying over-analytical techniques and experimental techniques in a converged asymptote [23].

\section{B. Smart Design and Packaging-an IoT Sensor Node}

Our framework reflects the key role of laboratory widely practiced techniques over a wide range of non-invasive scanning scenarios, which is hitherto be explored, especially Machine Learning (ML) enabled engines on-chip or off-chip. The measurement ranges are often restricted by the lack of available analytical techniques, often unawareness and distinguishably resolved with off-the-shelf ML strong mining techniques $[8,9,10,24]$. We deem an optimal scenario on a typical Pareto wavefront subject to optimization and deployment constraints, thus naturally demands Phenomenal Analyses of Interest (PAoI). The turn-in for critically evaluated problem course converges at the cost-design time, the volume of the research team, added magnitude into knockon attempts, vulnerability to scanning laser, data missing to poor contrast for non-invasive ambient conditions. This work provides additional proficiency to the analytical experts as well as a theoretical scientist [25].

The intermediate logical steps in a phenomenal process are illustrated in Fig. 2 for complex specimens exposed to noninvasive laser scanning to quantify the proposed LoC environment. The flow methodology tackles hidden malpractices by binding strictly with RBS indicators, discouraging thumb rule experiences, and adhering to effective and reliable pathways. Traditional practices are useful when offline training is required to achieve a converged objective function to mostly tailoring instrumentation techniques and yet reducing the complexity imposed frequently when non-linear systems are taken into account [26], [27].

The pre-simulation logs for $\mathrm{LoC}$ are used to resolve such discrepancies generating both qualitative and quantitative 
indicators generally restrained for complex specimens as exposed to non-invasive laser actuated instruments [28]. We also presented hybrid techniques that complement the analytical approaches, though logical in comprehension yet slow down the convergence to ToD without sacrificing costdesign time constraints [29], [30].

\section{MATERIAL AND MethodS}

In contrast, Fig. 2 provide a roadmap to a designer in answering the following questions:

- Identify the ultimate aim contribute a significant confidence interval to the aimed objective?

- The most likely confident neighborhood to find such a solution?

- What is the time to converge in meeting ToD constraints?

- Which techniques are suitable to satisfy empirical data and close adherence to analytical models?

- What is the cost-design time neighborhood bracket to satisfy non-invasive specimen constraints?

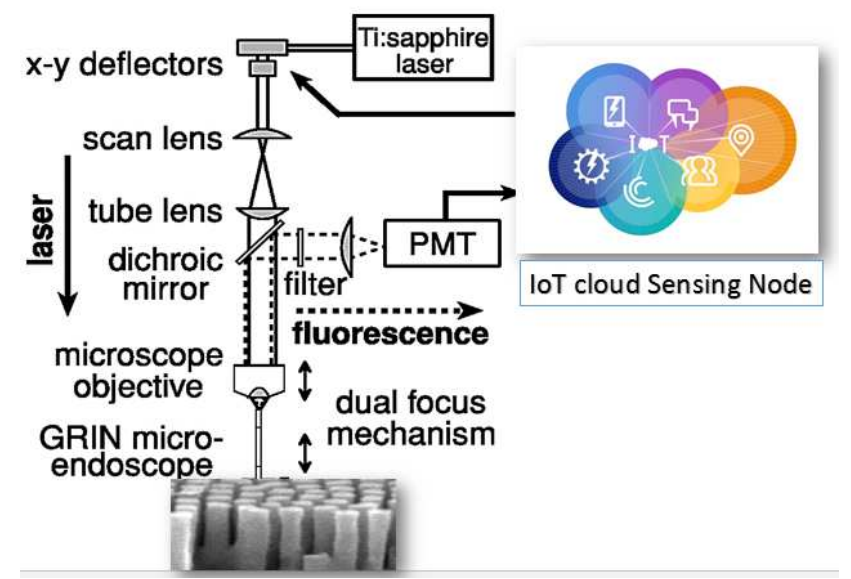

Fig. 2 Functional Viewpoint of Confocal Microscopy IoT Sensing Node

\section{A. Confocal Self Calibrated Interferometry based Laser Sensor (ConLSCI)}

The evaluation of the questions above is only done at the critical per view of a laboratory experiment while other factors of expertise, duration, budget, and off-the-shelf components availability are intact. Only an open solution would satisfy any multivariate constrained objective function but prone to capture np-hard state or local minima (a nonoptimal design parametric scenario). Our scenario is strictly a laser actuated non-invasive scanning adhere to LoC accuracy for a mobile environment, suitable to equivalent precision but much faster response depicted in Fig. 3. Such conflicting objectives often lead to less accuracy through zero-time field operations, most unlikely in traditional laboratory practices having a strong foundation on pedantic methodologies [31].

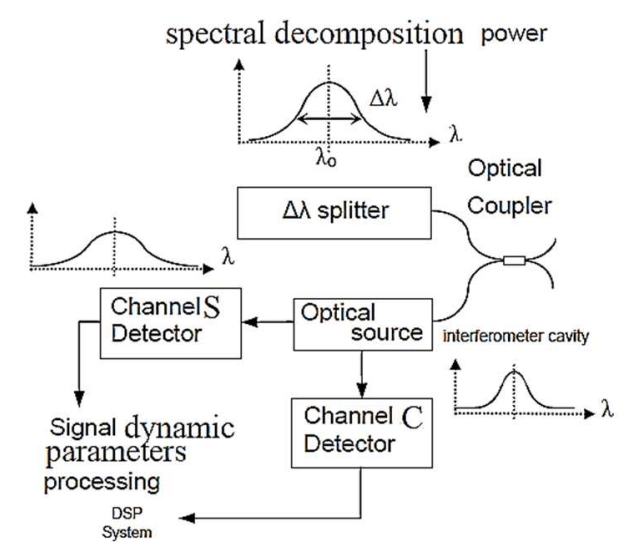

Fig. 3 Simplified view of ConLSCI sensor in Confocal

Smart instruments in any IoT network-sensing node are rapid-pace, self-adjustable to perform multiple scanning sessions in parallel on-demand. Only AI-enabled, fast cores provide such automated measurements, presumed to be selfcalibrated over a precise time, due to its visibility to Always Available Always Online (A3O) connectivity at IoT node.

\section{B. Phenomenal Analyses of Interest-The Specimen Constraints}

RBS plays a key role in aligning the diversity of technologies across the manufacturing process, yielding miniaturization of multi-functional scalability on a single core [32]. The relative approaches promise to provide OEM various choices to conform to their non-invasive scanning scenarios but require a robust measurement indicator profile, which can only be achieved in calibrating the sensitivity to a specific standard. As long as macro object illuminated specimens are in the account, the correct geometrical feature extraction is close to the actual specimen, but that is hard to achieve for microorganisms, hence give rise to an intense need for generic measuring resolution standards, especially in the wider deployment across the geographically spread labs and mobile profiles, to name a few.

The relative error of measurement is a precursor to questions raised on finding 'an optimal' techniques to satisfy both analytical and empirical neighborhood of confidence, as raised in 'what type of methodology to choose?' Here, the accumulative factors of interest lead to a concentrated design, which practically work across the scientist to reproduce and verify relative findings to both selective and specific ambient conditions.

A wider range of sensitivities above, correct, robust, and precise measurement can only be referenced when the frequency, analysis speed instantiation for a laser exposed specimen has almost closer numbers for LoC either in a laboratory environment, remotely operated units, or handheld IoT hubs. While keeping considerable grace to prohibition on the per recurring specimen cost, the accuracy of the result cannot be ignored at all, especially while an epidemic is monitored in non-favorable field situations. The concentration range of specimen arises to a significantly higher number but needs to be addressed when looking at a large canvass for geographical spread such as CovId19 nowadays [33], [34]. 


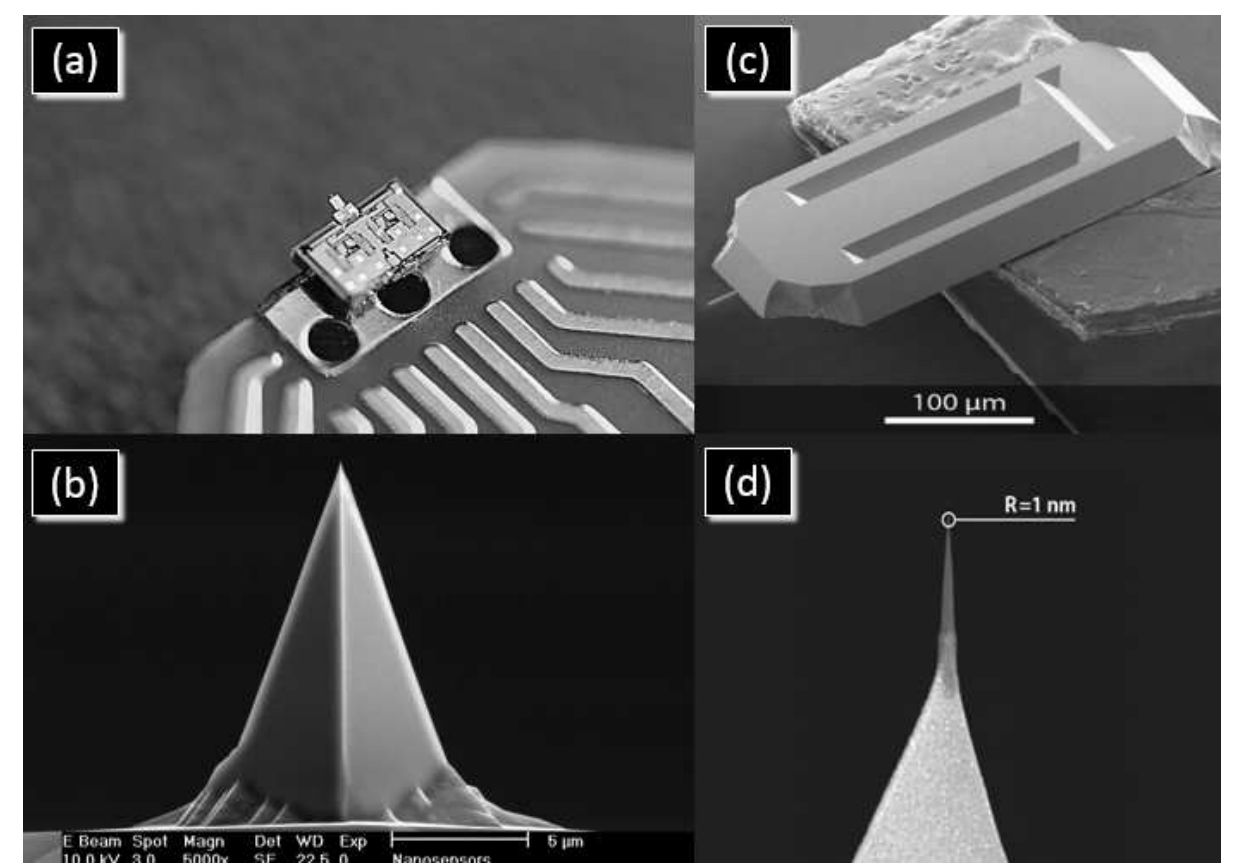

Fig. 4 Laser tip profile for high resolution and extra reinforcement hollow tube. (a). Single MEMS chip coarse apart augments sub-angstrom accuracy at lowest light leakage (b). Highly resonant, reflex coating compliments the plug and fit alignment (c). Tetrahedral tip at the end of the cantilever for correct ROI on the specimen (d). At the gold-coated silicon etched probe, resistive to reflectivity in the air well defined extra tip of carbon spike diamond.

The relative error of measurement is a precursor to questions raised on finding 'an optimal' techniques to satisfy both analytical and empirical neighborhood of confidence, as raised in 'what type of methodology to choose?' Here, the accumulative factors of interest lead to a concentrated design, which practically work across the scientist to reproduce and verify relative findings to both selective and specific ambient conditions.

While keeping considerable grace to prohibition on the per recurring specimen cost, the accuracy of the result cannot be ignored at all, especially while an epidemic is monitored in non-favorable field situations. Where concentration range of specimen arise to a significantly higher number but need to be addressed when looking at a large canvass for geographical spread such as CovId19 nowadays [34], [35].

\section{Laser Sensor Fabrication Profile}

The pivot role of RBS in aligning diversity of technologies across the labor-centric capita, the automatic well-integrated instrumentation cost per specimen though is heavy, but economically feasible when recurring over a long duration of field-testing. The notion of 'automatic and well-integrated testing environment' provides significant aid to the analytical model development later in labs and marking disease geography in affected areas [35], [36]. Analytical capability embedded in the IoT hubs brings a computational power with huge over the cloud storage assist composition of specimen characterization, interest sifting, required ambient conditions, and non-invasive workout.

There are four parts of non-invasive scanning devices largely found in analytical laboratories. They are the Function generator, illumination source, sensor part, analytical part. A unified division of these parts becomes challenging when it spreads across material engineering, chemical engineering, molecular biology, pharmacokinetics, or other pure sciences. These discrete functional elements would be the source, specimen, illuminator, sensor, and output [37], [38].

Unified description of instruments. This proposes that analytical instruments are comprised of five distinct modules: 1.Source 2. Sample, 3. Discriminator, 4. Detector 5. Output devise. We aim for Confocal Self Calibrated Interferometry based Laser Sensor (ConLSCI) to enable IoT maneuverability at any reasonably adjusted scenario. In order to let our approach to rendering its objectivity, one must have prior knowledge about the proper illuminating laser, various types of detectors, and projected output films or media. The accumulative interaction of radiation and specimen produces a series of compound observations revealing the type, quality, magnitude, volume, and quantity that yield a precise structure, later termed an analytical model. The multiple layer detectors produce a separate scan plane to render the specimen identity, nomenclature, structural details, and activity at microorganism scale. The compound observations contain a significantly higher precision of structural illumination produce in conjunction with multiple sets of detectors.

Section followed, elaborates detection, evaluation, surface elucidation on a molecular scale concentration on spectroscopic instruments in general and ConLSCI specifically.

\section{Design and Fabrication}

1) Instrumentation development and structure: Applications for light microscopy exist mainly in the biological arena. Material engineering has brought forth another dimension to the Nanoscale examination of the surface structure in a materials specimen $[39,40]$. They are widely used in compound analysis to distinguish lattice structures or to erode effects where the flow of information such as digital data is the traffic, e.g., electrode or chip surfaces that have been coated, to name a few.

2) Confocal spectrum measurement: We broke down the confocal microscopy in terms of functional viewpoint to 
extract qualitative features as depicted in Fig. 5. a typical set of features distinguish qualitatively the topography, i.e., the geometry of the surface of the object and apparent chromatic contours, morphological attributes which comprehend the magnitude, volume and pattern of nano-objects or atoms composing the subject itself and elemental proportion (a well maintained integrated lattice structure defined quantitative distribution of various constituents elements). The set of qualitative and quantitative information is obtained on the nanoscale of laser illumination, where specimen observation remains non-invasive to maintain presumptuously integrity of specimen geometry as well as life.

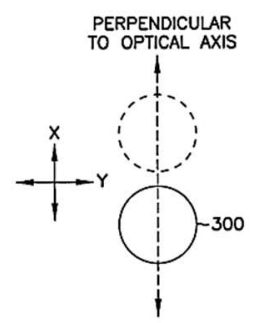

(a)

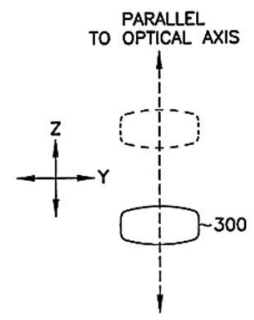

(b)

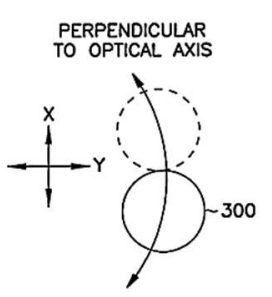

(c)

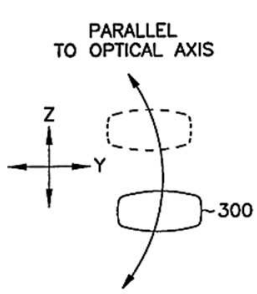

(d)

Fig. 5 Fabry-Prerot Cavity Impact on 3-D Coherence of Reference Channel. a), c) are orthogonal projection to illuminated axis, b), d) are Parallel to optical or illuminated axis.

Quantitative information may be possible using counting methods based on grids. The depth of visualization is threedimensional and high contrast over greater DPIs resolution recorded nondestructively at the confocal microscopes equipped with laser non-invasive scanning for the distinguished regular patterns in cells or tissues. Thick specimens are usually hard to image due to blurring, required an aggressive depth field control (at various orthogonal axis) with multiple sessions at the optics zone of detector and illumination. Our ConLSCI breakdown the successive microscopy session in confocal to achieve high contrast three dimensional images with high resolution obtained mostly depth field control along the perpendicular and parallel optical axis. Thus, reduce the inherent fading or turbulent noise may be an outcome of equipment vibration or specimen instability without compromising on laser source stabilized platform. ConLSCI confocal iterates various segmentation to draw clear planes among multi-dimensional reasonably solid specimen of tissues or cells.

The laser scanning on LoC rendering is completely nondestructive in nature to the structural or chemical hazards that may be induced when invasive techniques are deployed. Thick objects are viewed as successive recorded thin planes of laser imaging, hence keeping the underlying specimen's integrity intact. Multi-plane images are aligned with the clear objective of confocal microscopy in a contrast-enhanced specimen where fluorescent is of particular interest to mark the unknown patterns at a higher resolution, revealing the desired features for a particular pattern. Fluorescent techniques in our laser actuated framework extracts the thin natural patterns and derivatively approach to conform a desired analytical model. The process is augmented to accommodate the ultraviolet illumination spectrum in microscopy wherever two-photon instrumentation is adapted. The living cells, tissues, surface or skins, and diverse biological specimens need a deeper fined resolution by deep illuminated and detector stages.

3) Instrumentation development and structure: To meet high resolution and high SNR requirements, a high-power source is of great importance. The laser light source used in the system is a $1550 \mathrm{~nm}$ edge-emitting LED (E2LED) which can launch a maximum power of $50 \sim 60 \mu \mathrm{W}$ into its singlemode fiber pigtail.

Other optical components are thus required to work at 1550 nm. Fig. 5 is the I-P characteristic, and Fig. 5 (b) and (d) is the response of the LED, both at $25^{\circ} \mathrm{C}$. The most important parameters of the E2LED are its output power and the Central Wavelength (CWL). The output power is essential to the system resolution and should be as high as possible. The CWL will, when the light signal passes through the in-line filter (1551 nm CWDM filter), determine the narrowband signal intensity.

We observe that when the CWL matches the passband $(1551 \mathrm{~nm})$ of the in-line filter, the same variation will introduce a smaller error than when there is a mismatch. Therefore, the CWL should be around $1551 \mathrm{~nm}$. These two parameters change with temperature and driving current. The schematic of the laser pathway is derived in terms of standard instrumentation nomenclature to meet the requirement of offthe-shelf component availability, as follows:

- Source. Laser illumination is the source in the confocal microscope, where different resolution of planes is subject to specimen, which is close to the objective lens.

- Sample. Mounted slides hold the required sample of specimen, which is closer to the stage.

- Discriminator. The intermediary planes of highresolution images are detected by the laser illuminator tubes which multiply the photons to achieve higher intensity discrimination, readily distinguished in an analytical model. Discrimination is set on the primary lens, also called objective lens, whereas the detector is attached on the photo multiplier tubes.

- Detector. Photo multiplier tubes are the major player in detection phase which record a multi plane high resolution image with or without fluorescent derivatives to provide additional magnification at the EDA tools available on computer.

- Output: The manipulation of image formats among various compression or simply a raw image are readily available to standard computer display, where various tools can be deployed to highlight specific matter of 
interest, while taking into account the analytical models of known surface and structures of known cells or tissues from a database.

\section{RESULTS AND DisCUSSION}

The physical structure is key to the ConLSCI sensor, making it suitable for an IP-based sensor network, IoT to name it, both for laboratory desktop or portable instruments. Two orthogonally placed couplers control the sensor configuration in the proposed ConLSCI, usually made of optical fiber later transmit to a successive range of sensor cascaded to achieve desired resolution optically.

We are not considering any digital zooming at this stage, as they are mainly lossy and can be neutralized when engaged on desktop PCs. In this manner, source light illuminates laser actuated recording to cascade sets of sensors. A specific gap is maintained between the fiber at the input stage close to the objective lens and ongoing reflectors. A hollow photon multiplier glass tube provides a thermally fused onset to maintain the gap mentioned above, hence flexible to planes of input fiber and its cascade fused reflectors. Capillary tubes are thermally fused in the fiberglass to maintain predetermined gaps, which is not achievable for hard adhesive material, like epoxy. Organic materials have their issues when subject to mechanical vibration or hysteresis that induce turbulence or blurring effect when longer thermal effects damage the image with strong thermal spectrums.

At channel S, the input fiber face is exposed to illuminated reflection to the first incidental light at the end. Hollow glass tube allows reflected laser from cascade reflective surface also mentioned channel ' $C$ ' to propagate without any aberration. The laser detector at the end records the reflecting waves from the two crosses two orthogonal reflectors at the same stage found along the input area. Fiber interferometry issues are coped in associated sensors at ConLSCI. Low frequency induces the blurring effect in response to demodulation complexities and is resolved here by the auto healing network of IoT from time to time, calibrating and demodulating a given signal with standard accuracy. The calibration signal is an exact measurement for all the frequency dynamics in high-order transients. It is achieved with self-annealing attributes of auto-calibration instrumentation, especially in IoT sensor nodes. A noticeable interference exists when coherence is out of calibration usually in length due to the unequal length of the path inside an interferometer where two supporting legs are mounted.

At channel $\mathrm{S}$, the input fiber face is exposed to illuminated reflection to the first incidental light at the end. Hollow glass tube allows reflected laser from cascade reflective surface also mentioned channel ' $C$ ' to propagate without any aberration. The laser detector at the end records the reflecting waves from the two crosses two orthogonal reflectors at the same stage found along the input area. Fiber interferometry issues are coped in associated sensors at ConLSCI. Low frequency induces the blurring effect in response to demodulation complexities and is resolved here by an autohealing network of IoT from time to time, calibrate and demodulate a given signal with standard accuracy. The calibration signal is an exact measurement for all the frequency dynamics in high-order transients. It is achieved with self-annealing attributes of auto-calibration instrumentation, especially in IoT sensor nodes. A noticeable interference exists when coherence is out of calibration usually in length due to the unequal length of the path inside an interferometer where two supporting legs are mounted. As long as the difference is small than the interference threshold, magnified intensities coincide at the exact sum of constituent laser rays. We expose our methodology in ever-demanding interest in confocal microscopy for steerable fringe interference patterns.

It is an outcome of difference in exaggerated path length maintain sensor channel (Channel C) concurrent length in consistent with twice length in the hollow cavity as proposed in standard RBS indicators for Fabry-Perot gap length, also called cavity here, illustrated in Fig. 5. The fringe spectrum reflects any exceeded pathway in the Fabry-Perot cavity corresponds to asynchronous coherent or non-coherent reflected laser actuation. The absolute length of the Channel $\mathrm{S}$ is maintained as an indicator of absolute reference (also associated with calibration); in a way, it is always fixed to keep the pathway below the Fabry-Parot recommended cavity separation. The accumulative optical power of magnified dual reflections prohibits any interference or resultant infringes, thus setting the output power linear to the optical reference power.

However, vulnerability to fiber loss, dispersion edge loss, and power attenuation are hard to control for both channels. Unless compromised on cost-design time for high-end hollow tube choices leads to not a lucrative choice or when deployment scenarios are, for instance, an IoT network hub. Suppose we deem the thermal infusion of our sensor, the change in any temperature over a neighborhood of $4^{\circ} \mathrm{C}, 14^{\circ} \mathrm{C}$, $24^{\circ} \mathrm{C}$. As shown in Fig. 6, it depends on relevant indicators directly associated with the gap length in a photon multiplier tube gap.

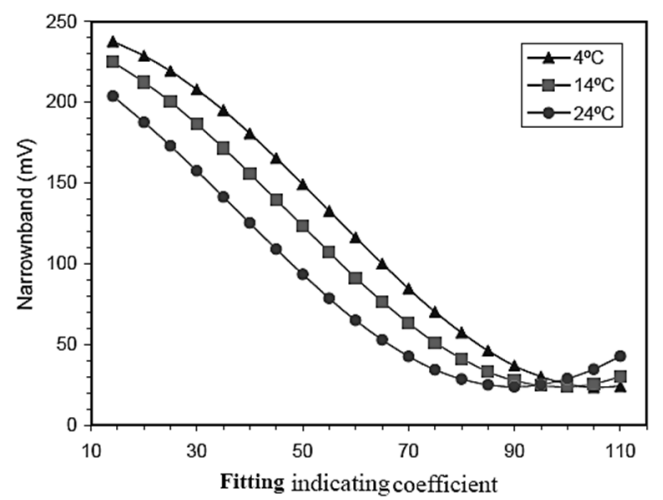

Fig. 6 Robustness Testing ConLSCI sensor at Narrowband Signals over Three Temperature Ranges

The laser E2LED tip in our nano scaling fabrication tested for favorable features as high accuracy $(0.1 \%$ typical error $)$ shown in Table I(a). A high speed $(10 \mathrm{MHz}$ full-power bandwidth), low distortion $(-80 \mathrm{dBc}$ from any input), low noise (94 dB SNR, $10 \mathrm{~Hz}$ to $20 \mathrm{KHz}$ and $70 \mathrm{~dB}$ SNR, $10 \mathrm{~Hz}$ to $10 \mathrm{MHz})$ as tabulated in Table $\mathrm{I}(\mathrm{b})$, and direct division mode (allowing the ratio of two channels to be obtained directly). Table I enlist noise robustness on both channel ' $\mathrm{S}$ ' and ' $\mathrm{C}$ ', termed as Channel I and Channel II for generic nomenclature. Readers are familiar with them as used in a monolithic real- 
time computational capturing loop that provides precision analog multiplication, division, and exponentiation.

TABLE I

NOISE ROBUSTNESS OF E2LED LASER TIP

\begin{tabular}{|c|c|c|c|}
\hline $\begin{array}{l}\text { a. Noi } \\
\text { gain) }\end{array}$ & $\begin{array}{l}\text { caused by dark curre } \\
\text { Channel I: 6.3mV@ } \\
\text { Channel II: 6.0mV@ }\end{array}$ & $\begin{array}{l}\text { nd electron } \\
2.9 \mathrm{mV} @ \\
2,2.7 \mathrm{mV} @\end{array}$ & $\begin{array}{l}\Gamma=19 \mathrm{oC}, \text { Full } \\
2\end{array}$ \\
\hline $\begin{array}{l}\text { b. DC } \\
\text { pigtail }\end{array}$ & ptical input (Full gain, & $\mathrm{M} \Omega$, input & ty from LED \\
\hline Seq & LED Driving Current & Channel I & Channel II \\
\hline No. & $(\mathrm{mA})$ & $(\mathrm{mV})$ & $(\mathrm{mV})$ \\
\hline 1 & 4 & 6.3 & 6.0 \\
\hline 2 & 8 & 6.3 & 6.0 \\
\hline 3 & 16 & 8.0 & 7.5 \\
\hline 4 & 24 & 11.0 & 10.7 \\
\hline $\begin{array}{l}\text { c. Cros } \\
\text { I) }\end{array}$ & talk (Full gain, Mod. In & $=0.1,500 \mathrm{nV}$ & ut to Channel \\
\hline Seq & Frequency $(\mathrm{KHz})$ & Channel I & Channel II \\
\hline No. & & $(\mathrm{mV})$ & $(\mathrm{mV})$ \\
\hline 1 & $\mathrm{DC}$ & 7.0 & 6.0 \\
\hline & 10 & 7.0 & 6.0 \\
\hline 2 & 50 & 7.0 & 6.0 \\
\hline 3 & 100 & 7.0 & 6.0 \\
\hline 4 & 150 & 7.1 & 6.1 \\
\hline
\end{tabular}

d. Offset adjustable range:

Channel I: $+77.9 \mathrm{mV} \sim-83.4 \mathrm{mV}$

Channel II: $+83.0 \mathrm{mV} \sim-101.2 \mathrm{mV}$

e. Temperature drift

No measurable temperature drift has been observed when the temperature of the preamplifiers is increased from $23^{\circ} \mathrm{C}$ to $45^{\circ} \mathrm{C}$.

It has wide input dynamic range, low error $(0.25 \%$ of reading possible), wide bandwidth $(400 \mathrm{kHz})$. The accuracy and bandwidth requirement for this E2LED project is $150 \mathrm{kHz}$, with a resolution of $0.01 \%(80 \mathrm{~dB})$; see Table I (c). The bandwidth of both dividers is more than sufficient to meet the project's requirements, but the noise performance will require significant improvement to meet the $0.01 \%$ resolution. One can argue here on how turbulence or fluctuations are catered in the mechanical fitting of two channels such that their ratio is constant, usually obtained canceling the channel ' $\mathrm{S}$ ' or

' $C$ ' gap out? In order to achieve the full range of the measurement for the desire optical intensity sensor probe is additionally designed where the processing of data and interpretation of results become consistent with the sensor sensitivity at the other end where the detector is mounted. A logarithmically linear relation is maintained at the interferometer to simplify the outcomes in the desired measurement range. We achieve it while fabricating capillary tube alignment with the laser actuated sensor, primarily an active source of non-invasive illumination at controlled geometrical parameters such as length, radius, surface area, breadth and elasticity strain, or stress modulus. Here, readers can imagine that this benefit is exploited due to the flexibility offered by the non-organic adhesive methodology. A straightforward sensor in our methodology is restricted to provide the best optical feature while maintaining noninvasive attributes intact for both specimen and framework. At the same time, secondary analysis for decomposition of output spectrum or counting of fringe in Channel $\mathrm{C}$ is a joint task for EDA and the hosting platform on a standard computer.
Spectrum reflects any exceeded pathway in the Fabry-Perot cavity corresponds to asynchronous coherent or non-coherent reflected laser actuation. The absolute length of the Channel $\mathrm{S}$ is maintained as an indicator of absolute reference (also associated with calibration); in a way, it is always fixed to keep the pathway below the Fabry-Parot recommended cavity separation. The accumulative optical power of magnified dual reflections prohibits any interference or resultant infringes, thus setting the output power linear to the optical reference power.

However, vulnerability to fiber loss, dispersion edge loss, and attenuation of power is hard to control for both channels, unless compromised on cost-design time for high-end hollow tube choices, which leads to not a lucrative choice when deployment scenarios are instance, an IoT network hub. If we deem the thermal infusion of our sensor, the change in any temperature over a neighborhood of $4^{\circ} \mathrm{C}, 14^{\circ} \mathrm{C}, 24^{\circ} \mathrm{C}$. As shown in Fig. 6, it depends on relevant indicators directly associated with the gap length in a photon multiplier tube gap.

The turbulence or fluctuations are catered in the mechanical fitting of two channels such that their ratio is constant, usually obtained canceling the channel ' $\mathrm{S}$ ' or ' $\mathrm{C}$ ' gap out. In order to achieve the full range of the measurement for the desire optical intensity sensor probe is additionally designed where the processing of data and interpretation of results become consistent with the sensor sensitivity at the other end where the detector is mounted. A logarithmically linear relation is maintained at the interferometer to simplify the outcomes in the desired measurement range. We achieve it while fabricating capillary tube alignment with the laser actuated sensor, primarily an active source of non-invasive illumination at controlled geometrical parameters such as length, radius, surface area, breadth and elasticity strain, or stress modulus. Here readers can well be imagined. This benefit is exploited due to flexibility offered by choice of nonorganic adhesive methodology. A straightforward sensor in our methodology is restricted to provide the best optical feature while maintaining non-invasive attributes intact for both specimen and framework. At the same time, secondary analysis for decomposition of output spectrum or counting of fringe in Channel $\mathrm{C}$ is a joint task for EDA and the hosting platform on a standard computer.

\section{A. Grating-Assisted Operating-Point Limitations}

The diffraction limit of ConLSCI prevents optical imaging beyond the micrometer scale. The ultra-miniaturization of near field optics is subjected to the confocal microscopy's objective lens and nullifies the diffraction of asynchronous reflected beams. We adopt a standard practice of 0.1 micrometer radial laser source, which not only reduces the limitations imposed on diffraction but is also equally mountable in a Fabry-Perot cavity as in our ConLSCI. The near field brings the laser source close to the field or specimen, increasing image dot per inch pixels for a narrow radial laser source.

L-V curves reciprocate similar to temperature drift in Table I (e) in Fig. 6 are at different temperatures. The driving current at which the LED has a CWL of $1551 \mathrm{~nm}$ can be determined for different temperatures. In the lower curve as intrinsically dependent on temperature, optimal working points are labeled by open circles, and the corresponding output power can thus 
be found. It can be seen that in order to have the highest power and the best CWL the ConLSCI sensor has to work under high temperature and a large driving current. However, both conditions may shorten the operating life of the ConLSCI sensor and E2LED. Currently, the working conditions are chosen to be $84 \mathrm{~mA}$ and $14{ }^{\circ} \mathrm{C}$, with an output power of 52 $\mu \mathrm{W}$.

\section{B. Laser Ablation Limitations}

Evanescent waves are attributed to refraction of light, which bends in the media of capillary tube tip, hence prone to laser beam leakage out of the sensor tip. This anomaly is corrected, bringing the specimen and the laser source in the closest vicinity, which allows adjustment of Petri plate for appropriate raster and creating a sharp, high-resolution NFOM in the near field of optical signal mapping fluorescent 3D image. A small amount of the light leaks out of the tip via the evanescent wave. Unlike NFOM, which particularly benefits cells and tissues image-planes capturing, the Scanned Near Field Optical Microscopy (SNFOM) yields far better resolution when exposed to observing a surface as finegrained as to reach $100 \mathrm{~nm}$. The excitation wavelength plays a key role, while nanoparticle visualization is recorded onto fluorescence segmentation needs to maintain emission properties compatible with the excitation system. In the scattering model for ambient light and fluorescent mode, the nanocarriers leakage induces large particles distributed equilibrium mostly in region $15 \mathrm{~nm}$ to $62 \mathrm{~nm}$. The leakage concentration is shown in Fig. 7 while ambient light or fluorescent scanning. Longer exposure and capture time prevent tracking of particles, especially at low concentrations, mainly due to fractional light leakage.

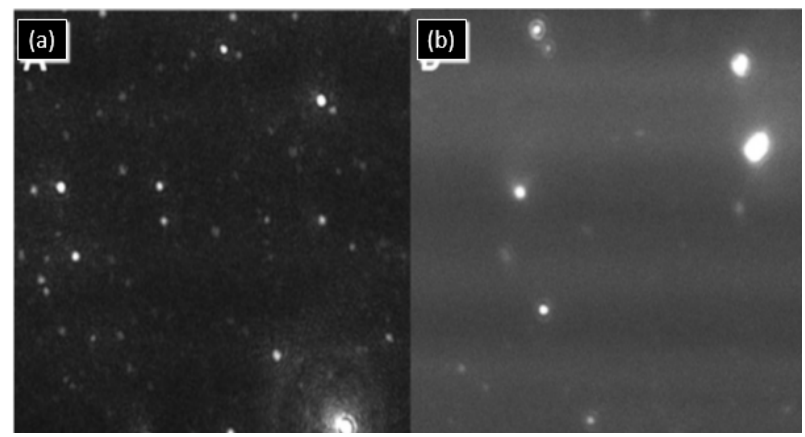

Fig. 7 Complex nanocarriers spectrum visualization for laser tip leakage in (a) scattering ambient light (b) scattering fluorescent segmentation.

The right dilution may is determined once we adjust both channel ' $\mathrm{S}$ ' and channel ' $\mathrm{C}$ ' at the confocal hollow tube on a cascade reflectors eliminating high pass filters as shown in Fig. 7 (b). Large topographic scans when subject to low concentration nanoparticles contain the bound components of leakage need to be adjusted with two tubes shown in Fig. 2; their distribution before and after the relative encapsulation is measured differently in both ambient laser and fluorescent light depicted in Fig. 8.

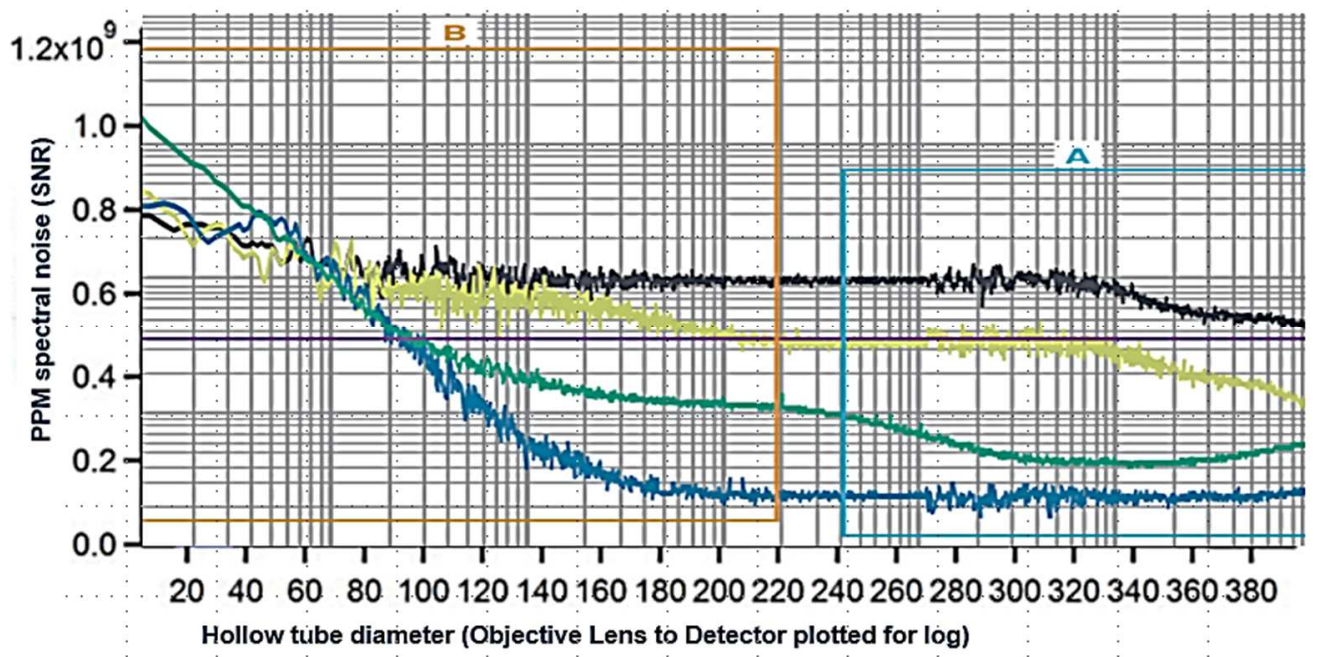

Fig. 8 Hollow PMT spectral distribution at differential fluorescent light scattering as a fraction of total non-bound nanoparticles tip leakage.

The encapsulation for parts per million particles over the leakage spectrum is called signal to noise ration mentioned along the vertical axis. Effective encapsulation of hollow tube diameter is strictly a function of laser actuated channel length and the objective lens to detector end. Whereas various sample-specific structures, probe tip scattering flexibility (due to non-polished edges) significantly contribute to nanoparticles formation spectra, recorded at analog to a digital module or camera CCD resulting enhanced resolution image. This phenomenon is similar to scanning luminesce regular microscope imaging, where leaking effects are manually removed at the data acquisition block by aggregating or interpolating techniques such as down sampling. But these operations induce another noise, called quantization noise, prevalent in most digital processing signals. We reduce this by incremental imaging in various bitplanes and projecting on the CCD without any optical or digital processing, thus provide a large canvass of possible analytic operation at the researcher desk. Unlike [27], [41], 42], we brought forth and developed fluorescent segmented microscopy in achieving the following objectives:

1) First, prior knowledge of the sample provides flexibility in marking the fluorescence to the Region of Interest (ROI) at laser actuated probes without sacrificing the potential leakage incidences, as mentioned above. 
2) Second, deeper structural penetration is achieved in imaging biological entities as cells, tissues, or geometrical features vide their UV spectrum or two-photon scattered illumination at the laser actuated probe tip.

3) Third, similar to UV spectrometer where deuterium or tungsten illuminate the cuvette specimen or flow cells in a monochromatic ambiance, the detector at PMT and data acquisition boards have a similar configuration as shown in our ConLSCI as shown in Fig. 2. Hence results are comparable for significant contrast and our contribution to work.

4) Fourth, laser actuation in our probe is non-adhesively mounted, enabling its yield over a large spectrum of cells, tissues, microorganisms, material lattice structure, nanasurface analytical modeling.

The ever-growing demand for higher resolution bit plane imaging in microorganisms is restrained by the fine pitched laser actuated probe mounting or materials. These limits are overcome with a thermally annealed wide range of $\mathrm{L}-\mathrm{V}$ curves, as shown in Fig. 6 across $1551 \mathrm{~nm}$ illumination, which yield superior, reliable, robust, and stable fluorescent highlights of specimen either a living cell or substrate doping computer chips.

\section{CONCLUSION}

In this work, we highlight the unprecedented paradigm shift towards smart and non-invasive instrumentation. Demand has amplified with IoT deployment-an intelligent remote sensing node integrated with comprehensive field lab test capacities, such as CovID19, to name a few. We discuss fabrication methodology while developing laser tip profile, design structure, specimen constraints successively. Testing phases are split into robustness for low power, high SNR taking rigorously laser ablation limitations as well as gratingassisted operating limitations. We expose our decision indicators for laser actuation point of care detection (i.e., a sample located at a laboratory or remote smart hubs) in the Instrument-in-Loop (IiL) to facilitate better Time-toDeployment $(\mathrm{ToD})$ for a given scenario. Using the miniaturization technology to $7 \mathrm{~nm}$ scale, the fabricated laser tip encourages us to tailor the demand in a non-invasive instrumentation, widely used in scanning microorganisms in vitro and ex vitro experiments. It is expected to add great value to researchers engaged in smart remote monitoring such as living cells, tissues, and material nanometer topographical scanning.

We observe that the visual vulnerability specimen for Confocal Microscopy is contributed due to its large 3-D visual spectrum to extract topographical, morphological, and composite features. The rastered back and forth 3-D imaging technique enables Confocal Microscopy for its widest application in biological and material sciences and hence the rose challenge of predicting large missing or incorrect data obtained during experiments. It is common observation while capturing the best geometrical ROI features. The laser draws a high current along at a significantly higher temperature, leading to shorter laser tip life.

The E2LED tip in our ConLSCI exhibits longer operational hours while subject to typically $84 \mathrm{~mA}$ and $14{ }^{\circ} \mathrm{C}$, with an output power of $52 \mu \mathrm{W}$. It enables a large window of resilience to luminescence propagation disturbance, e.g., where deuterium or tungsten illuminate the cuvette specimen or flow cells in a monochromatic ambiance the detector at both PMT and data acquisition boards. Additional reinforcement is achieved while exposing the capillarycompact fabrication of the sensor warmed to $\mathrm{CO} 2$ laser and set to a reasonably pointed edge for the compatible mount. The silver-coated reflective surface becomes the major intensity lobe which reflects completely internal as in other optical microscopic instruments, electron microscope to name. Researchers are expected to rely on a wider range of sensitivities above, correctness, robustness, and precise measurement. They can only be referred to when the frequency, analysis speed instantiation for a laser-exposed specimen has almost closer numbers for LoC. For the field experimentalists LOC may be a laboratory environment, remotely operated units, or handheld IoT hubs. We encourage technologists to adopt the ConLSCI framework suitable to smart instruments in any IoT network sensing node that are rapid-pace, self-adjustable to perform multiple scanning sessions in parallel on-demand.

\section{REFERENCES}

[1] P. Arthasarathy, P. Tamarapu and S. Vivekanandan, “A typical IoT architecture-based regular monitoring of arthritis disease using time wrapping algorithm." International Journal of Computers and Applications 42 (2020): 222-232.

[2] K. Amanli, A. Seyit, "A 3D imaging and visualization workflow, using confocal microscopy and advanced image processing for brachyuran crab larvae." Journal of Microscopy 266 (2017): 307-323.

[3] Z. Huang, X. Ling, "Synchronous detection of vascular tension and nitric oxide release in pulmonary artery: A combined application of confocal wire myograph with confocal laser scanning microscopy." Vascular (2020): 1708538120917555.

[4] K. E. McCracken, J. Yoon, "Recent approaches for optical smartphone sensing in resource limited settings: a brief review," Anal. Methods, 8(36), pp. 6591-6601, Sep. 2016.

[5] N. Z. Azeemi, "Handling Architecture-Application Dynamic Behavior in Set-top Box Applications," 2006 International Conference on Information and Automation, Shandong, 2006, pp. 195-200.

[6] F. Giancarlo, L. Fotia, F. Messina, D. Rosaci, M. L. Sarné, “A meritocratic trust-based group formation in an IoT environment for smart cities." (2020).

[7] Z. Wuchao, T. Wang, Y. Gan, J. Yang, H. Zhu, A. Wang, Y. Wang W. $\mathrm{Xi}$, "Effect of micropore/microsphere topography and a siliconincorporating modified titanium plate surface on the adhesion and osteogenic differentiation of BMSCs." Artificial cells, nanomedicine, and biotechnology 481 (2020), pp. 230-241.

[8] Qualcomm ${ }^{\circledR}$ Snapdragon ${ }^{\mathrm{TM}} 865$ mobile platform scales $5 \mathrm{G}$ and leading 5th gen AI to power next generation https://www.qualcomm.com/products/snapdragon-865-5g-mobileplatform (Retrieved Mar 2020).

[9] The Qualcomm Snapdragon Tech Summit reveals breakthroughs in 5G, AI, XR, and PCs https://www.youtube.com/watch?v=VCpIoXQxW0 (Retrieved April 2020).

[10] B. Paul, M. Janssen, P. Herder, "The dual effects of the Internet of Things (IoT): A systematic review of the benefits and risks of IoT adoption by organizations.” Int. J. Inf. Manag. 51 (2020): 101952.

[11] S. Sebastian, R. Louise, J. Meyer, "Confocal microscopy imaging of the biofilm matrix.” Journal of microbiological methods 138 (2017): pp. $50-59$.

[12] M. Ghazanfar, S. A. Shah, "IoT-Flock: An Open-source Framework for IoT Traffic Generation." ArXiv abs/2004.00844 (2020).

[13] N. Z. Azeemi, G. Al-Utaibi, O. Al-Basheer, "Customer-in-Loop Adaptive Supply Chain Migration Model to Enable IoT", IJITEE, ISSN: 2278-3075, Volume-9 Issue-6, pp. 1755-1762, April 2020.

[14] F. Faezeh, M. Sayad, H. A. Jolfaei, M. Alazab, “Artificial Intelligence for Detection, Estimation, and Compensation of Malicious Attacks in 
Nonlinear Cyber-Physical Systems and Industrial IoT." IEEE Transactions on Industrial Informatics 16 (2020): 2716-2725.

[15] Q. Mei, H. Jing, Y. Li, W. Yisibashaer, J. Chen, B. N. Li, Y. Zhang, "Smartphone based visual and quantitative assays on upconversional paper sensor," Biosens. Bioelectron, 75, pp. 427-432, Jan. 2016.

[16] G. Wu, X. Di, “Towards Distributed SDN: Mobility Management and Flow Scheduling in Software Defined Urban IoT." IEEE Transactions on Parallel and Distributed Systems 31 (2020): pp. 1400-1418.

[17] D. Sione, E. Jones, R. J. Harvey, J. M. Skousen, T. J. Schoenherr, "Designing hardware for the boundary condition round Robin Challenge," Kansas City National Security Campus, Sandia National Laboratories (2017).

[18] J. V. Capella, A. Bonastre, R. Ors, M. Peris, "A wireless sensor network approach for distributed in-line chemical analysis of water," Talanta, 80(5), pp. 1789-1798, Mar. 2010.

[19] R. Cheikhousman, M. Zude, D. J. R. Bouveresse, C. L. Leger, D. N. Rutledge, and I. B. Aragon, "Fluorescence spectroscopy for monitoring deterioration of extra virgin olive oil during heating," Anal. Bioanal. Chem., 382(6), pp. 1438-1443, Jul. 2005.

[20] E. L. Johan, J. A. Abuellil, A. C. Reyes, M. Abouzied, S. Yoon and E. S. Sinencio, "A Fully Integrated Maximum Power Tracking Combiner for Energy Harvesting IoT Applications." IEEE Transactions on Industrial Electronics 67 (2020): 2744-2754.

[21] K. Ojha, T. Ankita. "Chapter 19 Nanomaterials for removal of waterborne pathogens opportunities and challenges." Waterborne Pathogens (2020).

[22] N. Ramanujam, "Fluorescence spectroscopy in vivo," in Encyclopedia of Analytical Chemistry, John Wiley and Sons Ltd. Chichester, 2000; pp. 20-56.

[23] N. Z. Azeemi, Z. Hayat, G. Al-Utaibi, O. Al-Basheer, "Hybrid Data Protection Framework to Enhance A2O Functionality in Production Database Virtualization”, IGRTE, Volume-8 Issue-6, pp. 5691-5697, Mar. 2020.

[24] D. Chen, W. Yang, J. Hu, Y. Cai, X. Tang, "Energy-efficient secure transmission design for the internet of things with an untrusted relay." IEEE Access 6, 11862-11870 (2018).

[25] N. Z. Azeemi, A. Sultan and A. A. Muhammad, "Parameterized Characterization of Bioinfomatics Workload on SIMD Architecture," 2006 International Conference on Information and Automation, Shandong, 2006, pp. 189-194.

[26] P. Deshmukh, S. Solanke, Review paper: sarcasm detection and observing user behavioral. Int. J. Comput. Appl. 166 (2017)

[27] T. Tanwar, S. Tyagi, S. Kumar, "The Role of internet of things and smart grid for the development of a smart city, in Intelligent Communication and Computational Technologies," LNNS, vol. 19, ed. by Y. Hu, S. Tiwari, K. Mishra, M. Trivedi (Springer, Singapore, 2018), pp. 23-33.

[28] A. Kumar, S. Bharti, "Design and performance analysis of OFDM and FBMC modulation techniques.” Sci. Bull. Electr. Eng. Fac. 17, 30-34 (2017).

[29] N. Z. Azeemi, "Exploiting Parallelism for Energy Efficient Source Code High Performance Computing," 2006 IEEE International Conference on Industrial Technology, Mumbai, 2006, pp. 2741-2746.
[30] N. Khan, M. Alsaqer, H. Shah, G. Badsha, A. Abbasi, S. Salehian, "The $10 \mathrm{Vs}$, issues and challenges of big data," in International Conference on Big Data and Education (ACM, New York, 2018), pp. 52-56.

[31] R. Karimian, A. Nashmil, P. Hashemi, A. K. Jamosh, A. Afkhami, H. Bagheri. "The Principles and Recent Applications of Bioelectrocatalysis." (2020).

[32] W. Z. Khan, Y. Xiang, M. Y Aalsalem, and Q. Arshad, "Mobile phone sensing systems: a survey," IEEE Comm. Surveys \& Tutorials, 15(1), pp. 402-427, Jan. 2013.

[33] S. Yu, W. Xiao, Q. Fu, Z. Wu, C. Yao, H. Shen, and Y. Tang, “A portable chromium ion detection system based on a smartphone readout device," Anal. Methods, 8(38), pp. 6877-6882, Oct. 2016.

[34] L. Lixiang, Z. Yang, Z. Dang, C. Meng, J. Hao-tian, D. Wang, G. Chen, J. Zhang, H. Peng and Y. Shao. "Propagation analysis and prediction of the COVID-19." Infectious Disease Modelling 5 (2020): 282-292.

[35] "Coronavirus Disease 2019 (COVID-19)". Centers for Disease Control and Prevention. https://www.cdc.gov/coronavirus/2019ncov/symptoms-testing/testing.html (Retrieved March 2020).

[36] "Coronavirus disease (COVID-19) technical guidance: Laboratory testing for 2019-nCoV in humans". who.int https://www.who.int/emergencies/diseases/novel-coronavirus2019/technical-guidance/laboratory-guidance (Retrieved March 2020).

[37] Ocean Control, VIC, Australia, Smartphone electromagnetic sensor, [Online]. Available: https://oceancontrols.com.au/ESS-002.html. (Retrieved March 2020).

[38] Ocean Control, VIC, Australia, Smartphone UV sensor, [Online] Available: https://oceancontrols. com.au/ESS-001.html. (Retrieved March 2020).

[39] The Royal Swedish Academy of Sciences, Stockholm, Sweden, "Molecular Machines, Scientific Background on the Nobel Prize in Chemistry." 2016, https:/ www.nobelprize.org/nobel_prizes/chemistry/laureates/2016/advance d-chemistryprize2016.pdf (Retrieved Mar 2020).

[40] K. Wak, B. Okeon, J. Bae, "Integrated Design and Fabrication of a Conductive PDMS Sensor and Polypyrrole Actuator Composite.' IEEE Robotics and Automation Letters 5 (2020): 3758-3765.

[41] K. Yang, H. Peretz-Soroka, Y. Liu, and F. Lin, "Novel developments in mobile sensing based on the integration of microfluidic devices and smartphones," Lab Chip, 16(6), pp. 943-958, Mar. 2016.

[42] Malvern Instruments (2015) Manual: Nano-Sight NS300 user manual MAN0516. Malvern Instruments, Malvern Malvern-Instruments (2014) Nanosight NS300 NTA software guide. Malvern Instruments, Malvern, pp 1-24.

[43] S. Ehsan, B. Ghaemi, R. Sahraei, Z. M. Sabzevari, S. Kharrazi and A. Amani. "Colloidal synthesis of tunably luminescent AgInS-based/ZnS core/shell quantum dots as biocompatible nano-probe for high-contrast fluorescence bioimaging." Materials science \& engineering. C, Materials for biological applications 111 (2020). 